\title{
INFLUENCE OF POLYETHYLENE GLYCOL ADDITIVE ON PERFORMANCE OF POLYSULFONE AND POLYETHERSULFONE MEMBRANE
}

\author{
Siti Hawa Mohamad ${ }^{1,2 *}$, Hasan Zuhudi Abdullah ${ }^{1}$, Maizlinda Izwana Idris ${ }^{1}$, \\ Zawati Harun $^{1,2}$, Ahmad Fauzi Ismail ${ }^{3}$, Muhamad Zaini Yunos ${ }^{1,2}$, Sulaiman Hasan ${ }^{1}$ \\ ${ }^{1}$ Faculty of Mechanical and Manufacturing Engineering, \\ Universiti Tun Hussein Onn Malaysia, Batu Pahat 86400, Johor, Malaysia. \\ ${ }^{2}$ Advanced Materials and Manufacturing Centre (AMMC), \\ Faculty of Mechanical and Manufacturing Engineering, \\ Universiti Tun Hussein Onn Malaysia, \\ Batu Pahat 86400, Johor, Malaysia. \\ ${ }^{3}$ Advanced Membrane Technology Research Center (AMTEC), \\ Universiti Teknologi Malaysia, Skudai 81310 Johor Bahru, Malaysia. \\ Email: cthawamd@gmail.com
}

\begin{abstract}
This paper focuses on the performance of polysulfone (PSf) and polyethersulfone (PES) ultrafiltration membranes with polyethylene glycol (PEG). The aim of this study is to determine the effect of PEG as an additive in producing PSf and PES membrane. The flat sheet membranes were prepared via a phase inversion method in which the casting dope solution consists of polysulfone and polyethersulfone separately as polymer, while $\mathrm{N}$-methyl-2-pyrrolidone is used as the solvent material. PEG 400 was used as a pore forming additive in the casting dope solution. The morphology of membranes was analyzed by scanning electron microscopy. The performance of membranes was evaluated in terms of pure water flux (PWF) and humic acid rejection. The effect of different concentrations of PEG additive exhibits significant improvement on PSf and PES membrane performance. The results indicated that PES ultrafiltration membrane exhibits better performance in PWF than does PSf membrane. It was found that the pure water flux increases as the PEG concentration increases (0 to $8 \mathrm{wt} \%)$ in casting solution. As a result, the morphology of membranes prepared with a high concentration of PEG has a larger pore size. It can be concluded that PES ultrafiltration membrane consisting of PEG 400 yields an excellent increase in pure water flux compared to PSf membrane.
\end{abstract}

Keywords: Polysulfone; polyethersulfone; polyethylene glycol; pure water flux.

\section{INTRODUCTION}

Membrane technology has a unique place in many industrial and water management applications. Millions of preventable deaths in developing countries are due to microorganisms which come from polluted drinking water (Mulder, 1991). In order to solve this problem, an ultrafiltration (UF) process can be useful to remove contaminants from the polluted water. Generally, UF is considered as a very promising process for drinking water production because of its compactness, easy automation and high removal rate of turbidity, organic matter and virus. With pore diameters from $10 \mathrm{~A}$ to $1000 \mathrm{~A}$, it is usually defined as limited to membranes and UF is recognized as a low pressure 
membrane filtration process (Mohamed \& Atan, 2012; Mulder, 1991). The most widely used polymers for the UF preparation membrane are polysulfone (PSf) and polyethersulfone (PES). Generally, PSf and PES are characterized by $\mathrm{SO}_{2}$ linkages, which give them high strength. They are also rigid, tough thermoplastic with a glass transition temperature, $\mathrm{T}_{\mathrm{g}}$ of $180-250^{\circ} \mathrm{C}$ and excellent high temperature properties and chemical inertness (Seader \& Henley, 2006). The main disadvantage of polysulfone and polyethersulfone is their hydrophobicity, which leads to an apparent tendency to interact strongly with a variety of solutes and thus makes them prone to fouling (Cheryan, 1998). This problem can be overcome with some modifications to the membrane by adding some additive. (Shieh, Chung, Wang, Srinivasan, \& Paul, 2001) determined that polyethylene glycol (PEG), being hydrophilic in nature, can be used to improve membrane selectivity and as a pore forming agent. Previous studies showed that the addition of PEG acts as a pore forming agent and also affects the thermodynamics and kinetics of the phase inversion process. (Kim \& Lee, 1998) systematically studied the effect of PEG on membrane formation by phase inversion. Their study showed that by increasing the ratio of PEG additive to solvent NMP, the casting solution becomes thermodynamically less stable. The membrane pore size becomes larger and the top layer becomes more porous. In this study, the effect of different concentrations of PEG 400 with polysulfone and polyethersulfone membrane on pure water flux, humic acid rejection and morphology are investigated and discussed in detail. Polyethylene glycol (PEG 400) was used as a pore forming additive to improve the permeability of the membrane. The membrane performance was evaluated using a cross-flow filtration method.

\section{EXPERIMENTAL}

\section{Materials}

Polysulfone and polyethersulfone were separately used as the base polymer in the membrane casting solution (Figure 1). PSf and PES were dried at a temperature of $60^{\circ} \mathrm{C}$ for 24 hours before use. N-menthyl-2-pyrrolidione (NMP) from Merck was used as solvent. Polyethylene glycol 400 (QRec) was used as an additive and pore forming agent in the dope solution. Distilled water was used as the main non-solvent in the coagulation bath for phase inversion purposes.
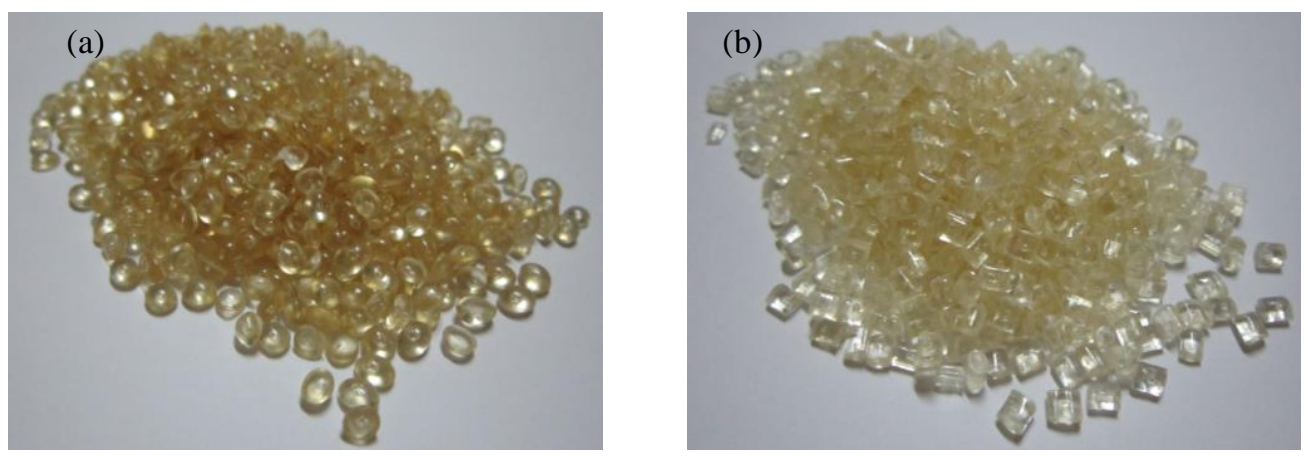

Figure 1. (a) Polyethersulfone and (b) polysulfone 


\section{Membrane Preparation}

PSf membrane and PES membrane were prepared using the phase inversion method. Casting solutions contained PSf and PES (separately), NMP and different concentrations of PEG 400 (0, 6 and $8 \mathrm{wt} \%$ ). The PSf and PES polymer were separately dissolved in NMP and stirred and heated at $60^{\circ} \mathrm{C}$ for several hours by a mechanical stirrer at $500 \mathrm{rpm}$ and at room temperature. Then, additive was added with continuous stirring until the dope solution was completely dissolved and homogeneous. Once completely homogeneous, the dope solution was kept in an ultrasonification machine for several hours to remove the air bubbles. The dope solution was poured onto a glass plate at room temperature and it was cast using a casting knife. After casting, the glass plate with the cast film was immersed in distilled water. The cast films changed color from transparent to white immediately after immersion into the coagulation bath. The membrane was washed and kept in distilled water for several hours. The flat sheets were air dried at room temperature for more than 24 hours before testing. Figure 2 shows polythersulfone membrane after immersion in distilled water.

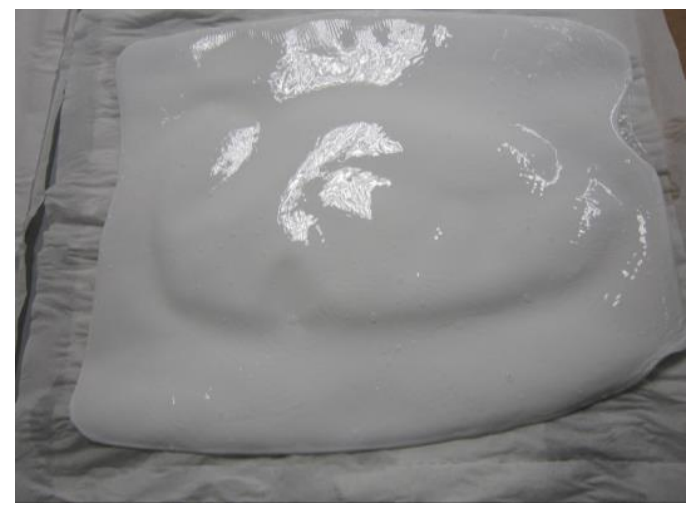

Figure 2. Polyethersulfone membrane after immersion in distilled water.

\section{Membrane Characterization}

Scanning electron microscopy (SEM) was used to examine the flat sheet membrane morphology. The membranes were cut into small pieces and were immersed in liquid nitrogen for several seconds. Then, the membrane samples were fractured carefully and coated with gold before testing.

\section{Pure Water Flux and Rejection}

The permeation flux and rejection of the prepared membranes were measured using an ultrafiltration cross-flow test at 3 bars. The flat sheet membrane sample was cut into a circle shape with an area of $2.376 \times 10^{-3} \mathrm{~m}^{2}$ and placed in the test cell with the active skin layer facing the incoming feed. The pure water flux experiments used distilled water as feed whereas the rejection experiments used humic acid. The volume of permeate was collected and measured. The membrane performance of pure water flux (PWF) for PSf and PES ultrafiltration membrane were calculated from Eq. as follows:

$$
P W F=Q /(A \times \Delta t)
$$


PWF in $\left(\mathrm{L} / \mathrm{m}^{2} \mathrm{~h}\right)$, where $\mathrm{Q}$ is the volume of permeate $(\mathrm{L}), \mathrm{A}$ is the membrane surface area $\left(\mathrm{m}^{2}\right)$ and $\Delta \mathrm{t}$ is the permeation time (h). Rejection was characterized with $100 \mathrm{mg} / \mathrm{L}$ humic acid after the PSf and PES membrane was filtered with distilled water. The concentrations of feed and permeate solution were determined using a UV spectrophotometer (Shimadzu UV-160) at a wavelength of $254 \mathrm{~nm}$ against a reagent blank. The rejection percentage was calculated using the following Eq. (2):

$$
R(\%)=\left[1-\left(C_{p} / C_{f}\right)\right] \times 100
$$

where $\mathrm{R}(\%)$ is the rejection percentage, $\mathrm{C}_{\mathrm{p}}$ is the concentration of permeates and $\mathrm{C}_{\mathrm{f}}$ is the concentration feed.

\section{RESULTS AND DISCUSSION}

\section{Morphology of PSf and PES Ultrafiltration Membrane}

The images of the PSf and PES ultrafiltration membranes generated by SEM are shown in Figure 3 and Figure 4 respectively. It can be observed that the PSf and PES membranes have an asymmetric porous structure which consists of a dense layer on the top of the membrane, an intermediate porous sub-layer and a sponge-like structure at the bottom surface layer. Based on Figure 3 and Figure 4, it is clear that by increasing the PEG concentration in the casting solution, the porous finger-like structure size at the top surface of the membrane is increased.

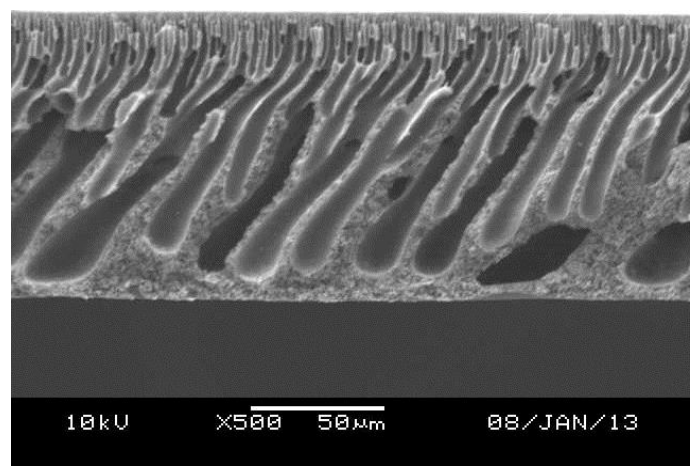

(a)

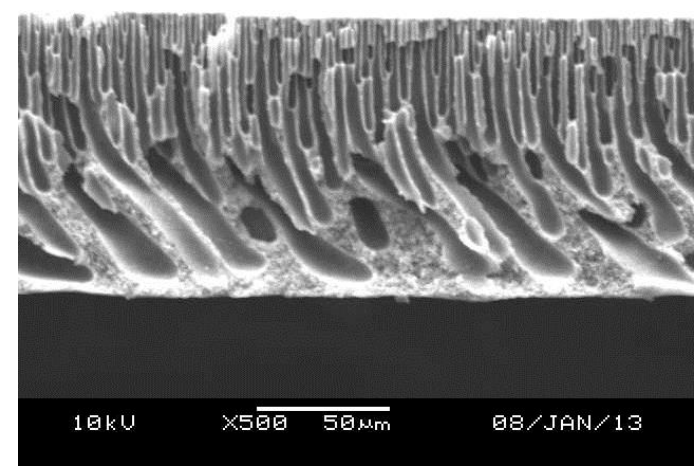

(b)

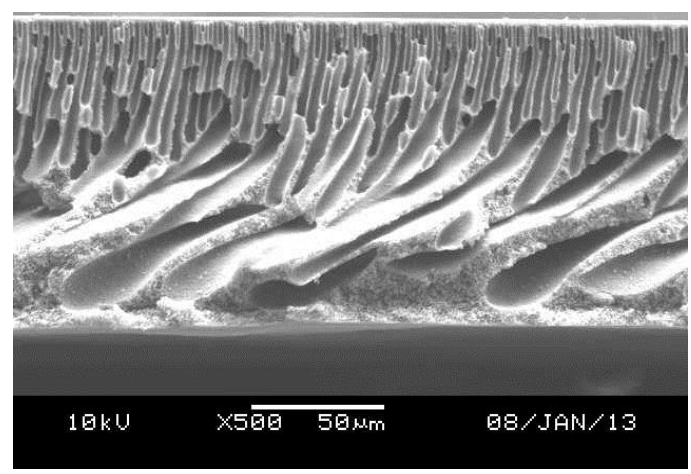

(c)

Figure 3. Cross-section of SEM images of PSf membrane with different concentrations of PEG: (a) $0 \mathrm{wt} \%$ PEG, (b) $6 \mathrm{wt} \%$ PEG (c) $8 \mathrm{wt} \%$ PEG 
Referring to a previous study (Idris, Mat Zain, \& Noordin, 2007), as the concentration is increased, the macrovoids increase in number and size, thus enhancing the formation of many finger-like pores of membrane. These figures also show the spongy bottom layer, which may be due to slow precipitation of the membrane during immersion in the coagulation bath after the casting process. Basically, in the phase inversion process, the formation of the membrane structure depends on the thermodynamic principles of the casting solution (Mulder, 1991).

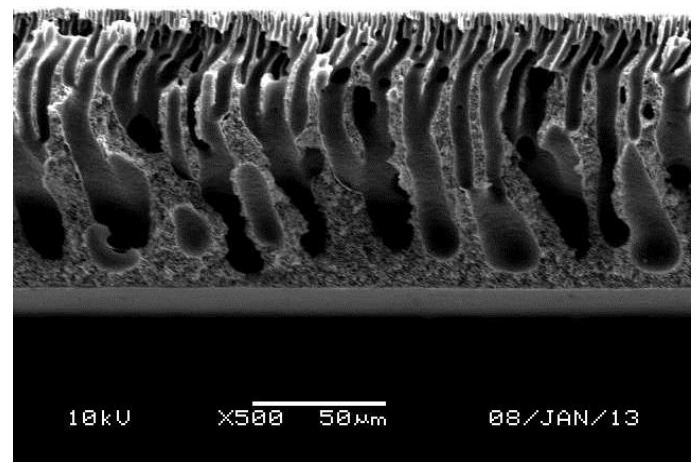

(a)

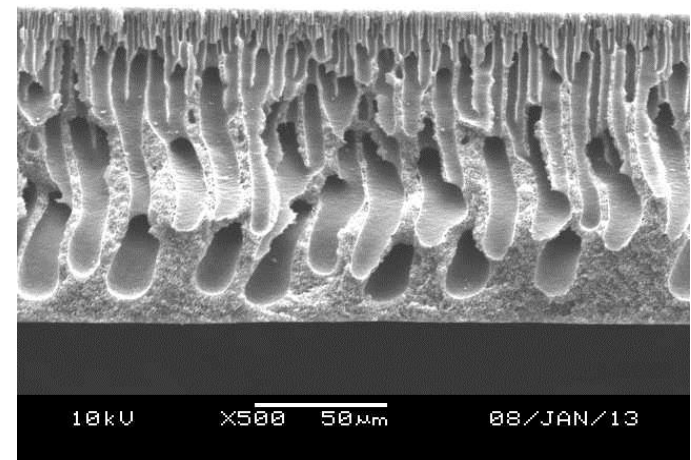

(b)

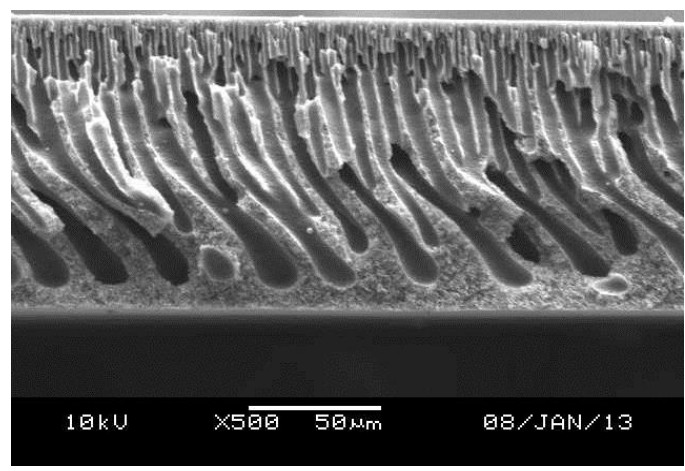

(c)

Figure 4. Cross-section of SEM images of PES membrane with different concentrations of PEG: (a) $0 \mathrm{wt} \%$ PEG, (b) $6 \mathrm{wt} \%$ PEG (c) $8 \mathrm{wt} \%$ PEG

\section{Pure Water Flux and Humic Acid Rejection}

Figure 5 clearly indicates that the pure water flux (PWF) performance of the PSf and PES membrane is significantly improved with an increasing percentage of polyethylene glycol (PEG 400) added to the casting solution. From Figure 5, the flux performance of PSf membrane increases as the percentage of PEG increases from $0 \mathrm{wt} \%\left(2.70 \mathrm{~L} / \mathrm{m}^{2} \mathrm{~h}\right)$ to 8 $\mathrm{wt} \%\left(80.81 \mathrm{~L} / \mathrm{m}^{2} \mathrm{~h}\right)$. The PES ultrafiltration membrane that contains polyethylene glycol as the additive has increased pure water permeation from $12.12 \mathrm{~L} / \mathrm{m}^{2} \mathrm{~h}$ to $113.64 \mathrm{~L} / \mathrm{m}^{2} \mathrm{~h}$, when the concentration of PEG increases from 0 to $8 \mathrm{wt} \%$. Based on pure water permeation, the PES membrane gives better performance in increasing the flux compared to the PSf membrane. This situation is due to the pore enhancement when the percentage of PEG increases in the casting solution. Based on previous studies, PEG acts as a pore forming agent to increase the pore structure of membrane (Shieh et al., 2001), since the PEG additive has hydrophilic properties, and therefore gives better interaction between the membrane surface and water permeation. Research by (Liu, Koops, \& Strathmann, 
2003) showed that PEG can be used to enhance the polymer which is PES solution viscosity and to enhance pore interconnectivity when added in appropriate amounts.

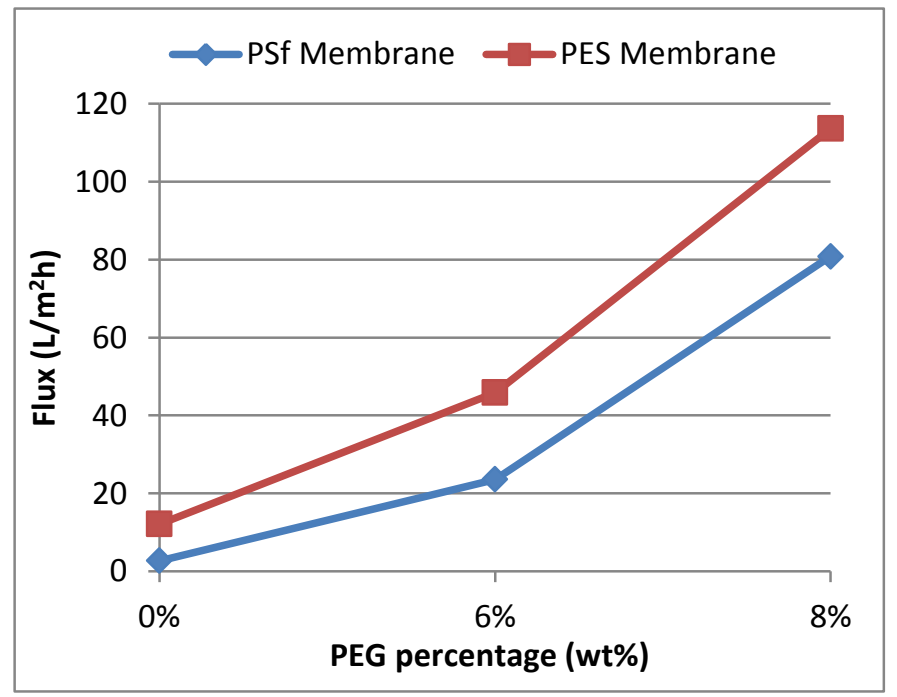

Figure 5. Pure water flux permeation for PSf and PES membrane

The effect of PEG 400 as an additive on the permeate flux and humic acid (HA) rejection on the PSf and PES membranes is clearly presented in Figure 6. Based on the bar graph plotted, the humic acid rejection performance of the PSf and PES ultrafiltration membrane is not significantly different. The highest rejection performance by the PSf and PES membrane is $98.5 \%$. Meanwhile, the permeate flux of both ultrafiltration membranes decreases compared to pure water flux observation. The humic acid permeation for the PSf and PES membrane increased as the percentage of PEG additive increased in the casting solution. As a result, the PSf membrane rises from $2.45 \mathrm{~L} / \mathrm{m}^{2} \mathrm{~h}$ to $62.70 \mathrm{~L} / \mathrm{m}^{2} \mathrm{~h}$ when the percentage of PEG additive is increased. The permeation flux of HA for the PES membrane rises from $11.62 \mathrm{~L} / \mathrm{m}^{2} \mathrm{~h}$ to $89.22 \mathrm{~L} / \mathrm{m}^{2} \mathrm{~h}$ as the PEG 400 increases.

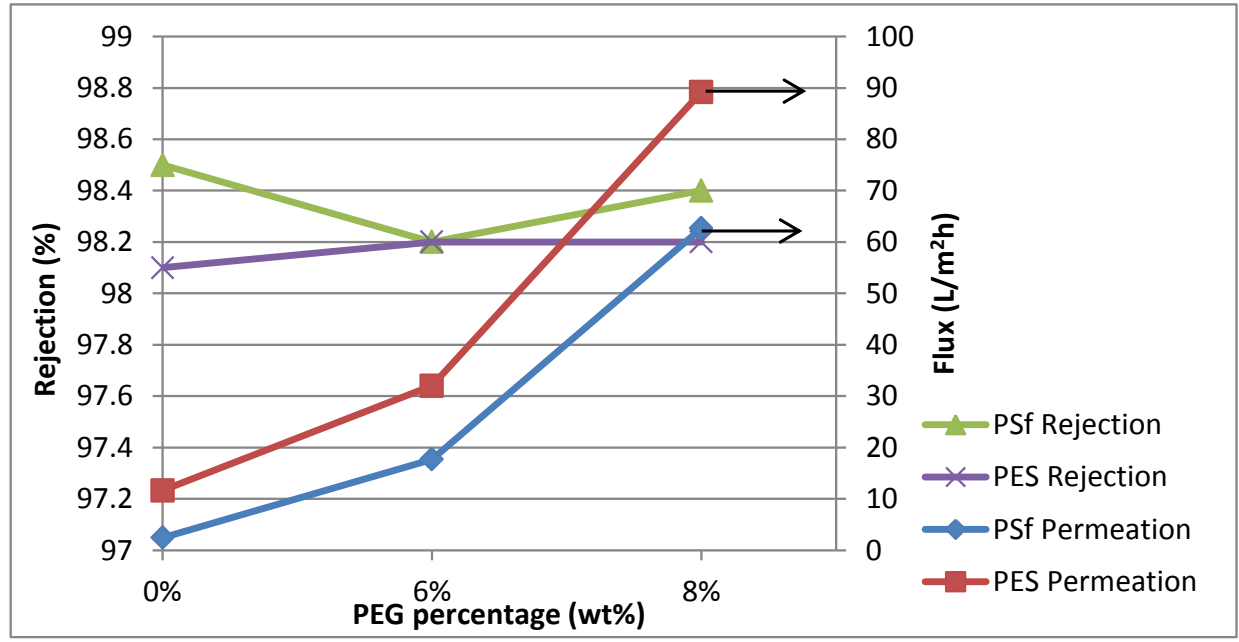

Figure 6. Humic acid permeation and rejection results for PSf and PES membrane. 


\section{CONCLUSION}

In conclusion, the presence of polyethylene glycol (PEG) of different concentrations as an additive has a significant effect on the performance and morphology of PSf and PES ultrafiltration membranes. The addition of different concentrations $(0 \mathrm{wt} \%, 6 \mathrm{wt} \%$ and 8 wt $\%$ ) of polyethylene glycol as an additive in the casting solution influences the morphology structure, pure water flux performance and humic acid rejection of the membranes. The PES ultrafiltration membrane consisting of PEG 400 shows an excellent increase in pure water flux compared to the PSf membrane.

\section{ACKNOWLEDGEMENTS}

The authors would like to thank the Ministry of High Education Malaysia and Universiti Tun Hussein Onn Malaysia for their Long Term Research Grant Scheme (LRGS vote A022) for support in providing the grant to implement the "High Performance of Polymeric Materials" project.

\section{REFERENCES}

Cheryan, M. (1998). Ultrafiltration and microfiltration handbook Boca Raton: CRC Publisher.

Idris, A., Mat Zain, N., \& Noordin, M. Y. (2007). Synthesis, characterization and performance of asymmetric polyethersulfone (pes) ultrafiltration membranes with polyethylene glycol of different molecular weights as additives. Desalination, 207(1-3), 324-339.

Kim, J. H., \& Lee, K. H. (1998). Effect of peg additive on membrane formation by phase inversion. Journal of Membrane Science, 138(2), 153-163.

Liu, Y., Koops, G. H., \& Strathmann, H. (2003). Characterization of morphology controlled polyethersulfone hollow fiber membranes by the addition of polyethylene glycol to the dope and bore liquid solution. Journal of Membrane Science, 223(1-2), 187-199.

Mohamed, W. A. N. W., \& Atan, R. (2012). Polymer electrolyte membrane fuel cell. International Journal of Automotive and Mechanical Engineering, 5, 648-659.

Mulder, M. (1991). Basic principles of membrane technology London: Kluwer Academic.

Seader, J. D., \& Henley, E. J. (2006). Separation process principles New York: John Wiley \& Sons.

Shieh, J. J., Chung, T. S., Wang, R., Srinivasan, M. P., \& Paul, D. R. (2001). Gas separation performance of poly(4-vinylpyridine)/polyetherimide composite hollow fibers. Journal of Membrane Science, 182(1-2), 111-123. 